\title{
Ceiling Culture-Derived Proliferative Adipocytes are A Possible Delivery Vehicle for Enzyme Replacement Therapy in Lecithin: Cholesterol Acyltransferase Deficiency
}

\author{
Masayuki Kuroda ${ }^{1,2,3, *}$, Yasuyuki Aoyagi ${ }^{1,2,3}$, Sakiyo Asada ${ }^{1,2,3}$, Hideaki Bujo ${ }^{2, *}$, Shigeaki Tanaka ${ }^{3}$, \\ Shunichi Konno ${ }^{3}$, Masami Tanio ${ }^{3}$, Itsuko Ishii ${ }^{4}$, Kazuhiko Machida ${ }^{5}$, Fumiaki Matsumoto ${ }^{6}$, \\ Kaneshige Satoh ${ }^{6}$, Masayuki Aso ${ }^{3}$ and Yasushi Saito ${ }^{7}$ \\ ${ }^{I}$ Center for Advanced Medicine, Chiba University Hospital, Chiba University, Chiba, Japan \\ ${ }^{2}$ Department of Genome Research and Clinical Application, Graduate School of Medicine, Chiba University, Chiba, Japan \\ ${ }^{3}$ CellGenTech, Inc., Chiba, Japan \\ ${ }^{4}$ Graduate School of Pharmaceutical Sciences, Chiba University, Chiba, Japan \\ ${ }^{5}$ Central Institute for Experimental Animals, Kawasaki, Japan \\ ${ }^{6}$ Department of Plastic and Reconstructive Surgery, Graduate School of Medicine, Chiba University, Chiba, Japan \\ ${ }^{7}$ Chiba University, Chiba, Japan
}

\begin{abstract}
Human proliferative adipocytes propagated via ceiling culture technique from subcutaneous fat tissue (designated as ccdPA) were herein evaluated for their potential as a recipient for retroviral vector-mediated gene transduction of a therapeutic protein delivery. Exposure to the ZsGreen-expressing vector supernatant using a cell preparation generated by a 7-day ceiling culture induced a 40-50\% transduction efficiency, with less than two integrated copies of viral genome per cell on average. The lcat gene-transduced human ccdPA secreted functional LCAT protein, correlating with the integrated copy number of vector genome. The gene-transduced cells could be expanded up to nearly $10^{12}$ cells from $1 \mathrm{~g}$ of fat tissue within one month after fat tissue preparation. The cells also maintained the potential to differentiate into adipocytes in vitro. The presence of human LCAT protein in serum was immunologically identified upon transplantation of lcatexpressing ccdPA into the adipose tissue of immune-deficient mice. These results indicated that human ccdPA has a novel therapeutic potential for LCAT-deficient patients. The clinical application in combination with cell transplantation shed a light on a development of a life-long protein replacement therapy for LCAT-deficient patients.
\end{abstract}

Keywords: Protein replacement therapy, lecithin:cholesterol acyltransferase, adipocyte, ceiling culture, gene therapy.

\section{INTRODUCTION}

The intriguing biology of pluripotent stem or progenitor cells has suggested the sustained production of therapeutic proteins to be a treatment for patients with serum protein deficiencies $[1,2]$. The ability of cells to self-renew at a high proliferation rate has led to the expectations that these cells are ideal targets for retroviral vector-mediated transgene delivery. Studies examining this concept have described the treatment of various diseases in animal models [3-10].

Lecithin:cholesterol acyltransferase (LCAT) is a plasma protein responsible for the conversion of plasma unesterified

\footnotetext{
*Address correspondence to these author at the Current address: Center for Advanced Medicine, Chiba University Hospital, Chiba University, 1-8-1, Inohana, Chuo-ku, Chiba, 260-0856, Japan; Tel: +81-43-222-7171, or +8143-441-4121; Fax: +81-43-226-8130; E-mail: kurodam@ @aculty.chiba-u.jp and Department of Genome Research and Clinical Application (M6), Graduate School of Medicine, Chiba University, 1-8-1, Inohana, Chuo-ku, Chiba, 260-8670, Japan; Tel: +81-432227171; Fax: +81-43-226-8130; E-mail: hbujo@faculty.chiba-u.jp
}

cholesterol into cholesteryl ester, and plays a central role in the formation and maturation of high-density lipoproteins (HDL), which are involved in reverse cholesterol transport. Genetic LCAT deficiencies have been identified, and more than forty different mutations have been identified to date (refer to HGMD: http://www.hgmd.cf.ac.uk/ac/index.php). Plasma LCAT is either absent or exhibits no catalytic activity in patients with a familial LCAT deficiency. Cholesteryl ester levels are markedly reduced in lipoproteins, abnormal cholesterol deposition is observed in the tissues of these patients, and patients often develop corneal opacity, anemia, proteinuria, and renal failure [11]. The efficacy of LCAT replacement therapy was shown by infusion of normal plasma [12, 13], but the effects were transient. In addition, replacement therapy with recombinant LCAT protein has not been established mainly because this is a rare condition, and due to the associated expenses for production of the recombinant protein. Therefore, life-long treatment with autologous cell-based therapy may contribute to the continuous replacement of enzymes. 
Recently, much attention has been paid to adipose tissue as a source of proliferative cells for cell-based gene therapy [14] and for regenerative therapy $[15,16]$. Two types of preparations have been reported to be sources of adiposetissue derived proliferative cells. One is stromal-vascular fractions (SVFs), which can be obtained as sediment by the centrifugation of collagenase-digested fat tissue $[17,18]$. The obtained cells are pluripotent and can differentiate to yield various cell types, including cardiomyocytes, chondrocytes, and osteoblasts, in addition to adipocytes [19]. The other cell preparation is obtained from the floating mature fat cell fraction of the centrifugation, followed by a ceiling culture [20]. The cultured cells maintain the ability to differentiate into mature adipocytes at a high frequency [10, 20, 21], and are presumably more committed to the adipocytes lineage.

In the present study, a target cell population was prepared from adipose tissue using the ceiling culture technique to develop a cell-based gene therapy of LCAT-deficient patients, and we designated the target cells as ceiling-culture derived proliferative adipocytes (ccdPA). The current study established this production procedure, and optimized the gene transduction conditions of human ccdPAs as therapeutic gene recipient cells. In addition, we assessed the capability and the safety of the lcat gene-transduced ccdPA as a LCAT-secreting device for protein replacement therapy. Therefore, we developed stable protein-producing human ccdPAs with self-renewing and high expansion capacities.

\section{MATERIALS AND METHODS}

\section{Construction of pCGThLCAT, A Retroviral Vector Plasmid Encoding the Human Lcat Gene}

The pDON-AI, Moloney Murine Leukemia virus (MoMLV) vector plasmid (TaKaRa Bio Inc., Shiga, Japan) was used as a recipient for the human lcat cDNA. The lcat cDNA was derived from total RNA prepared from HepG2 cells. The resulting cDNA was amplified by PCR using the following primer pair: 5'-ATCGGATCCAGGGCTGGAAA TGGGGCCGCCC-3' (forward) and 5'-ATCGGATCC GTCGACGGAAGGTCTTTATTCAGGAGGCGGGGG-3' (reverse). The forward primer contained a BamHI restriction site (underlined) and a Kozak sequence, and the reverse primer contained a SalI restriction site (underlined). The reverse primer also eliminated the polyA signal from the original lcat cDNA. The amplified PCR products were digested by BamHI and SalI and cloned into the corresponding sites of the pDON-AI plasmid. Thereafter, the neomycin resistant gene was removed by SalI and XhoI digestion and subsequent self-ligation, yielding the pCGThLCAT plasmid.

\section{Production of the Amphotropic Retroviral Vector}

The GMP grade retroviral vector CGT_hLCATRV was produced by TaKaRa Bio Inc. In brief, the pCGThLCAT vector was transfected into the ecotropic packaging cell line GP+E86 (ATCC\#: CRL-9642), and the supernatant was collected. The supernatant was used to infect the amphotropic packaging cell line GP+envAM-12 (ATCC\#: CRL-9641) to produce a master cell bank (MCB) for vector production. CGT_hLCATRV was prepared from culture supernatant of the MCB. The vector solution was aliquoted and stored at $-80^{\circ} \mathrm{C}$ until use. The vector titer was quantified by TaKaRa Bio Inc. using the One Step SYBR PrimeScript RT-PCR Kit with primer pairs from Retrovirus Titer Set (TaKaRa Bio Inc.). The ZsGreen-gene expressing retrovirus vector was similarly propagated.

\section{Cell Culture and Medium}

Dulbecco's modified Eagle's medium [DMEM]/F12HAM (Sigma-Aldrich, St. Louis, MO) and MesenPRO medium (Invitrogen, Carlsbad, CA) were used to maintain cultured cell lines. Fetal bovine serum (FBS) was purchased from SAFC Biosciences (Lenexa, KS). Cell passaging was performed twice a week.

\section{Isolation of cedPAs from Human Fat Tissue}

Subcutaneous adipose tissues were obtained from 16 healthy volunteers (C001-C016) with ages ranging from 19 to 42 years after informed consent was obtained with the approval and guidelines of the ethical committee at Chiba University School of Medicine, according to the Declaration of Helsinki. Ceiling culture techniques [20] were employed and optimized using C001-C012 fat tissues to isolate human ccdPAs as follows. Fat tissue was weighed, and each $1.0 \mathrm{~g}$ was digested with gentle agitation for $1 \mathrm{hr}$ at $37^{\circ} \mathrm{C}$ in $3 \mathrm{ml}$ of Hank's balanced salt solution (HBSS) containing $2 \mathrm{mg} / \mathrm{ml}$ collagenase (Collagenase NB 6 GMP Grade, SERVA, Heidelberg, Germany) and $40 \mu \mathrm{g} / \mathrm{ml}$ gentamicin (GENTACIN, Schering-Plough Co., Kenilworth, NJ). Thereafter, the solution was diluted with $10 \mathrm{ml}$ of DMEM/F12-HAM containing $20 \% \mathrm{FBS}$ and $40 \mu \mathrm{g} / \mathrm{ml}$ gentamicin (DMEM/FBS), mixed, and centrifuged at $400 \mathrm{x} \mathrm{g}$ for $1 \mathrm{~min}$. The pellet was removed as an SVF. The dilution steps were repeated 4 times to collect the floating cell fraction. The floating fraction was filtered with a 500- $\mu \mathrm{m}$ mesh (Netwell Insert, Corning Inc., Corning, NY) and seeded into flasks, which were filled with DMEM/FBS. After 7 days ceiling culture, cells that grew at the ceiling surfaces were harvested and seeded into flasks for the subsequent steps.

\section{Gene Transduction}

In preliminary experiments, the acceptability of the MoMLV vector for human ccdPA propagated in the course of ceiling culture revealed that longer culture times resulted in a higher resistance to retroviral vector transduction (data not shown). Therefore, the cells obtained by 7 day-ceiling culture were evaluated as a potential recipient for retroviral vector-mediated gene transduction. Human ccdPAs were seeded and incubated in DMEM/FBS at $37{ }^{\circ} \mathrm{C}$ for $24 \mathrm{hrs}$. Protamine sulfate (PS, Novo-Protamine Sulfate, $100 \mathrm{mg}$ for I.V. Injection, Mochida Pharm. Co. Tokyo, Japan) was used to optimize the transduction conditions $(0.5-16 \mu \mathrm{g} / \mathrm{ml})$. Gene transduction was performed in the presence of $20 \%$ FBS and $8 \mu \mathrm{g} / \mathrm{ml}$ PS at $37^{\circ} \mathrm{C}$ for $24 \mathrm{hrs}$. The viral vector concentration used for transduction was $2.0 \times 10^{9}$ RNA copies $/ \mathrm{ml}$, unless otherwise specified. After transduction, the medium was replaced with growth medium.

\section{Flow Cytometry}

Cells were suspended in phosphate buffered saline containing 2\% FBS (PBS/FBS). Fluorescein isocyanate (FITC) or phycoerythrin (PE)-conjugated antibodies were purchased 
from BD Farmingen (San Diego, CA) or Beckman Coulter (Fullerton, CA), or Ancell Corporation (Bayport, MN). Aliquots of cell suspensions $\left(4.5 \times 10^{4}\right.$ cells) were mixed with primary antibody in a total volume of $90 \mu \mathrm{l}$ and were incubated for $30 \mathrm{~min}$ at RT. The cell suspension was washed twice with PBS/FBS, and the cells were fixed in $200 \mu \mathrm{l}$ of PBS/FBS containing 1\% paraformaldehyde. Five thousand events were acquired for each antibody on a FACS Calibur apparatus using the CELLQuest acquisition software program (Becton, Dickinson and Company, Flanklin Lakes, NJ). ZsGreen expression was also examined in human ccdPAs. Non-transduced cells were used as a negative control.

\section{Quantification of Transduced Gene}

Genomic DNA was extracted from cultured cells and mouse adipose sections with the DNeasy Blood \& Tissue kit and the Gentra Puregene kit (QIAGEN, Hilden, Germany), respectively. The integrated vector copy number was quantified with the SYBR Premix Ex Taq (Perfect Real Time) kit (TaKaRa Bio Inc.). A known amount of pCGThLCAT DNA was used as a standard. The primer pairs were from the Retrovirus Titer Set (TaKaRa Bio Inc.). The DNA content in a human normal cell (6 pg/cell) [22] was used for calculating the average integrated copy number. Existence of transduced gene in transplanted adipose tissue was quantified with a TaqMan Gene Expression Master Mix (Applied Biosystems, Foster City, CA) using lcat-cDNA specific primers and probes designed by the Probe Finder Software program (Roche Diagnostics, Mannheim, Germany). All the real-time PCR reactions were performed using the ABI7500 Real-time PCR system (Applied Biosystems).

\section{Detection of LCAT Protein}

Culture medium and mice sera were diluted to a volume of $500 \mu \mathrm{l}$ with ice-cold phosphate buffered saline containing $0.2 \%$ Nonidet P-40 (PBS-NP40) and were incubated with 2.5 $\mu l$ of anti-LCAT rabbit monoclonal antibody (EPITOMICS, Burlingame, CA) for $18 \mathrm{hrs}$ at $4{ }^{\circ} \mathrm{C}$ with gentle rotation. Twenty micro-liters of TrueBlot anti-Rabbit Ig IP Beads (eBioscience, San Diego, CA) was added and incubated with rotation for $2 \mathrm{hrs}$ at $4{ }^{\circ} \mathrm{C}$. Bound proteins were pelleted by centrifugation, washed with PBS-NP40, and eluted by boiling in $10 \mu \mathrm{l}$ of $2 \mathrm{X}$ Laemmli's sample buffer. Immunoprecipitated samples were subjected to immunoblotting. Purified human LCAT (Roar Biomedical, Inc., New York, NY) or human plasma HDL (Calbiochem, Merck, Darmstadt, Germany) was used as a standard. An anti-LCAT rabbit polyclonal antibody (Novus Biologicals, Littleton, CO) and TrueBlot anti-Rabbit IgG HRP (1:5000) (eBioscience) were used as primary and secondary antibodies, respectively. The signals were detected with the SuperSignal West Femto Maximum Sensitivity Substrate (Thermo Fisher Scientific Inc.) and the LAS1000 apparatus (FUJI film, Tokyo, Japan).

\section{Measurement of LCAT Activity}

The procedure described by Ishii et al. [23] was modified to prepare the liposome substrate for the LCAT analyses. Two hundred microliters of $\left[{ }^{3} \mathrm{H}\right]$-cholesterol (American Radiolabeled Chemicals, Inc., St. Louis, MO) were evaporated to dryness by flushing $\mathrm{N}_{2}$ gas, and $5 \mathrm{ml}$ of the substrate mixture of Anasolv LCAT kit (SEKISUI MEDICAL Co. Tokyo, Japan) was added. The solution was sonicated with a Digital Sonifier Model 250 (BRANSON, Danbury, CT) at an amplitude of $40 \%$ and 0.5 second pulse cycles for $1 \mathrm{~min}$ a total of six times in an ice bath. The sonicated mixture was centrifuged at 3,000 rpm and stored at $4{ }^{\circ} \mathrm{C}$ until use. The reaction mixture contained $100 \mu$ l of labeled substrate, 4.5 $\mathrm{mM} \beta$-mercaptoethanol, $36 \mu \mathrm{g}$ of apolipoprotein A1 (Athens Research \& Technology, Athens, GA), and $100 \mu$ l of culture medium in a total volume of $220 \mu \mathrm{l}$. The reaction was performed at $37^{\circ} \mathrm{C}$ for $1 \mathrm{hr}$, and was terminated by the addition of $1.6 \mathrm{ml}$ of chloroform/methanol (2:1). One hundred microliters of water was added, and the organic phase was obtained by centrifugation. Fifty microliters of the organic phase was spotted onto Whatman flexible thin layer chromatography (TLC) plates (Whatman plc, Kent, UK). Samplespotted plates were developed with standards of cholesterol and cholesterol oleate in a glass tank using a solvent mixture of hexane/ethyl ether/acetic acid (146:50:4) by TLC. Developed TLC plates were air-dried and stained with iodine (Wako Pure Chemicals, Osaka, Japan). Cholesteryl ester spots were excised, and the radioactivity was determined by liquid scintillation spectrometry.

\section{Adipogenic Differentiation Assay}

Human ccdPA ( $3.5 \times 10^{4}$ cells) were seeded into BioCoat Collagen I 48-well Multiwell Plates (BD Biosciences) and grown to confluency over 3 days. Differentiation was induced with the PGM Bullet Kit (Lonza, Basel, Switzerland), and the cells were incubated for 2 weeks. The cells were fixed in $4 \%$ paraformaldehyde, washed twice with PBS, incubated with $60 \%$ isopropanol for $1 \mathrm{~min}$, and stained with Oil Red O solution (Chemicon International, Inc. Temecula, CA) for $20 \mathrm{~min}$. The accumulation of triglycerides was examined to confirm adipogenic differentiation using the Triglyceride E-test kit (Wako Pure Chemicals) according to the manufacturer's instructions. The protein content of the lysate was also determined with the Quick Start Bradford Dye Reagent (Bio-Rad Laboratories Inc.).

\section{Clonality Analysis by Southern Blotting}

Abnormal amplification of specific cell clones resulting from the integration of the retroviral vector genomic sequence was examined by Southern blotting according to the DIG (Digoxigenin) protocol (Roche Diagnostics). Genomic DNA extracted with the Gentra Pure Gene kit (QIAGEN) was digested with HindIII (Roche Diagnostics). Digested DNA $(6 \mu \mathrm{g})$ was subjected to agarose gel electrophoresis, followed by capillary transfer to a positively-charged nylon membrane (Roche Diagnostics). Human lcat cDNA was used as a template to synthesize probes by the PCR DIG Probe Synthesis kit (Roche Diagnostics). Hybridization was performed at $50{ }^{\circ} \mathrm{C}$ overnight. The membrane was washed and reacted with Anti-digoxigenin-AP, Fab fragments (Roche Diagnostics). The signals were detected using CDPStar with the LAS1000 apparatus (FUJI film). As positive control, 293 (European Collection of Cell Cultures) cells were transduced with a neomycin-resistant gene-containing version of the lcat-expressing retroviral vector, and typical single copy-integrated clones were selected. 


\section{Colony Formation Assay by Soft-Agar Containing Medium}

Anchorage-independent colony formation was examined by the soft-agar assay using the CytoSelect 96-well Cell Transformation Assay kit (Cell Biolabs, Inc., San Diego, CA). Ten thousand gene-transduced human ccdPAs were seeded into 96-well plates in triplicates along with 100, 1000, or 10,000 HeLa cells (European Collection of Cell Cultures) as a positive control.

\section{Monitoring Human LCAT Secretion in Mouse Model}

Animal experiments were performed in the Central Institute for Experimental Animals (CIEA, Kanagawa, Japan) according to the Ethical Guidelines for Animal Experimentation from CIEA to examine the delivery of LCAT protein in vivo. To identify the transplanted cells, cells were stained using the PKH26 Red Fluorescent Cell Linker kit for General Cell Membrane Labeling (Sigma-Aldrich) one passage prior to transplantation. Expanded cells were harvested, washed with Ringer solution containing $0.5 \%$ human serum albumin (HSA, Benesis Corp. Osaka, Japan) four times, and re-suspended to a final cell concentration of $3 \times 10^{7}$ cells $/ \mathrm{ml}$. The cell suspension $(50 \mu \mathrm{l})$ was injected into the adipose tissue between the shoulder-blades of NOD/Shi-scid IL$2 \mathrm{R} \gamma^{\text {null }}$ (NOG) mice [24]. Buffer alone was injected as a control. All mice were bred in a vinyl-isolator and six animals were sacrificed to collect serum samples at each time point (Day 1, and at 1, 3, and 6 months). Six and three animals were used for the transplanted and control groups, respectively, for each time point. The transplanted region was taken using fluorescent microscopy on a SZX16 reflected fluorescence system (OLYMPUS corp. Tokyo, Japan), and sections were frozen at $-80{ }^{\circ} \mathrm{C}$ until use.

\section{Statistical Analysis}

Data are presented as means \pm S.D. Statistical comparison were made by Student's $t$-test or by ANOVA followed by the post hoc Dunnett or Tukey test using the SPSS software program. The integrated copy number, positive rate, and LCAT activity were analyzed to determine whether there was a linear correlation between these variables. For this analysis, we calculated a linear correlation coefficient (Pearson $r$ value) and the corresponding P-value (two tailed) based on these assumptions. In all cases, P-values of less than 0.05 were considered to be statistically significant.

\section{RESULTS}

\section{Preparation of Gene-Transduced Human ccdPAs}

The optimization of cell-processing steps was carried out with fat tissues obtained from 16 healthy volunteers (C001C016). Adipose tissue-derived proliferative cells were assessed for their suitability in ceiling culture, gene transduction, and cell expansion, using two culture media, DMEM/ F12-HAM supplemented with 20\% FBS (DMEM/FBS) and MesenPRO medium, respectively. The ceiling culture was performed in DMEM/FBS in comparison to MesenPRO medium. The cell yield of $\mathrm{C} 012$ after the ceiling culture from $1 \mathrm{~g}$ adipose tissue was $7.1 \times 10^{5} \pm 1.0 \times 10^{5}$ and $2.1 \times 10^{5} \pm$ $0.2 \times 10^{5}$ cells in DMEM/FBS and MesenPRO medium, respectively, showing that a higher cell yield was obtained in DMEM/FBS than in MesenPRO medium $(\mathrm{p}<0.05)$. The flow cytometric analyses showed that cells in DMEM/FBS tended to be homogeneous in shape and size, in comparison to those grown in MesenPRO medium (Fig. 1a). The gene transduction of the cells after the ceiling culture was next assessed using the two medium types. The above cells which were frozen after ceiling culture (C010) in DMEM/FBS were recovered, incubated for 4 days, and seeded for gene transduction in MesenPRO medium or DMEM/FBS medium. After transduction with the lcat-expressing retroviral vector, the cells were passaged several times in the respective medium, and cell samples were subjected to copy number quantification 12 days after transduction. DMEM/FBS was more effective than MesenPRO medium for the gene transduction of human ccdPAs when a retroviral vector was employed under the appropriate conditions (0.94 \pm 0.10 copies/cell vs. $0.36 \pm 0.09$ copies/cell, $\mathrm{p}<0.05)$. Finally, the effects of the
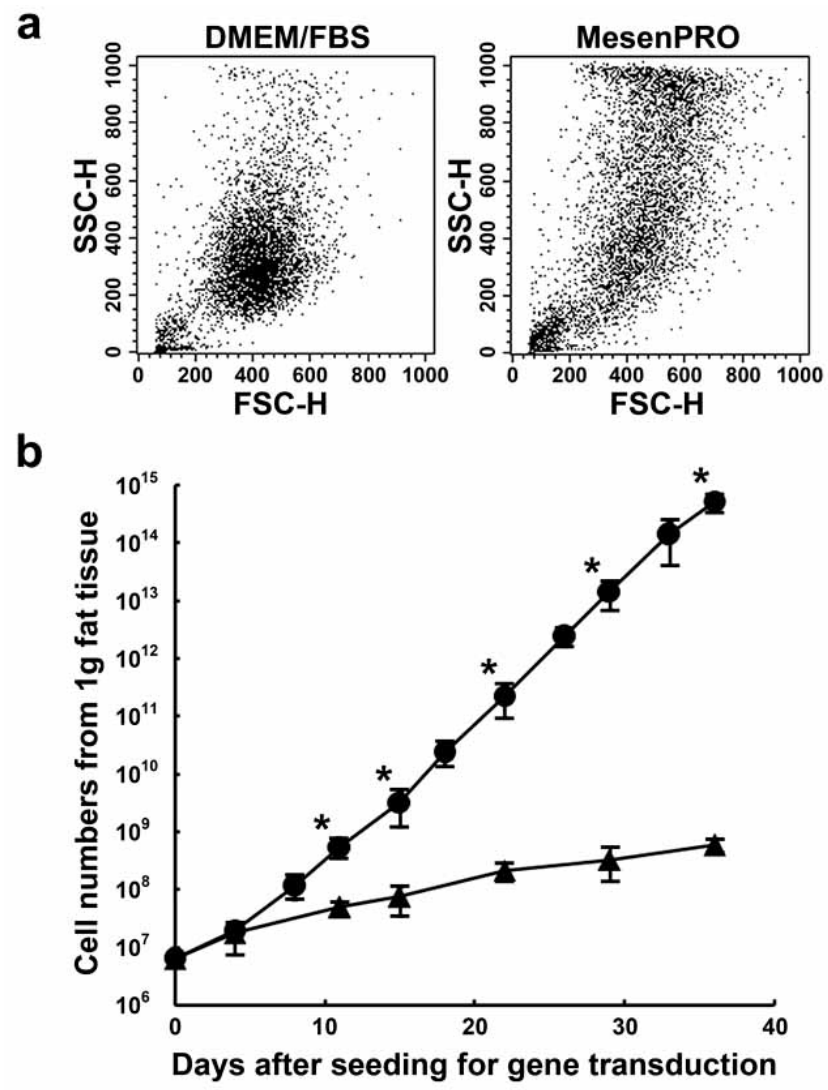

Fig. (1). Comparison of DMEM/FBS and MesenPRO media for the preparation of human ccdPAs. (a) The cells (C012) prepared by ceiling culture in DMEM/FBS (left panel) or MesenPRO medium (right panel) were subjected to a FACS analysis. The dot-plot (forward-scattered vs. side-scattered) of both cell populations are shown. A representative plot is shown for each medium. (b) The cells derived from $\mathrm{C} 013$ were used for expansion. Cell numbers were counted during proliferation for 35 days in DMEM/FBS (closed triangle) or MesenPRO medium (closed circle) after gene transduction in DMEM/FBS. Cell numbers are presented from $1 \mathrm{~g}$ of fat tissue. Data are presented as the mean \pm SD $(n=3)$. *p<0.05 vs. MesenPRO medium at each day after seeding. 
incubation media on the gene-transduced cell expansion were examined in $\mathrm{C} 013$ cells. The doubling times of the cells in the MesenPRO medium were significantly shorter than those in DMEM/FBS $(31.7 \pm 4.8$ hours vs. $119.4 \pm 29.6$ hours, $\mathrm{p}<0.05)$. The transduced cell number expanded to more than $3 \times 10^{4}$ fold of the original number in a month when grown in MesenPRO medium (Fig. 1b). Therefore, DMEM/FBS was chosen for the ceiling culture and gene transduction, and the MesenPRO medium for cell expansion of ccdPA, respectively, in subsequent experiments.

\section{Characterization of Human ccdPAs}

The cell surface antigen profile was analyzed by FACS for human ccdPAs (Fig. 2a). The populations of $\mathrm{CD}^{-} 1^{-}$/ $\mathrm{CD} 45^{-}$cells were significantly increased in the ccdPA preparation, in comparison to SVF-derived cells $(99.1 \pm 0.3 \%$ vs. $95.6 \pm 0.1 \%, \mathrm{p}<0.05)$, indicating that ceiling culture technique excludes CD31-positive and/or CD45-positive cell populations in comparison with cells prepared from SVF. The ccdPAs were positive for CD9, CD10, CD13, CD29, CD44, CD59, CD90, CD105, CD146, and HLA-ABC, and
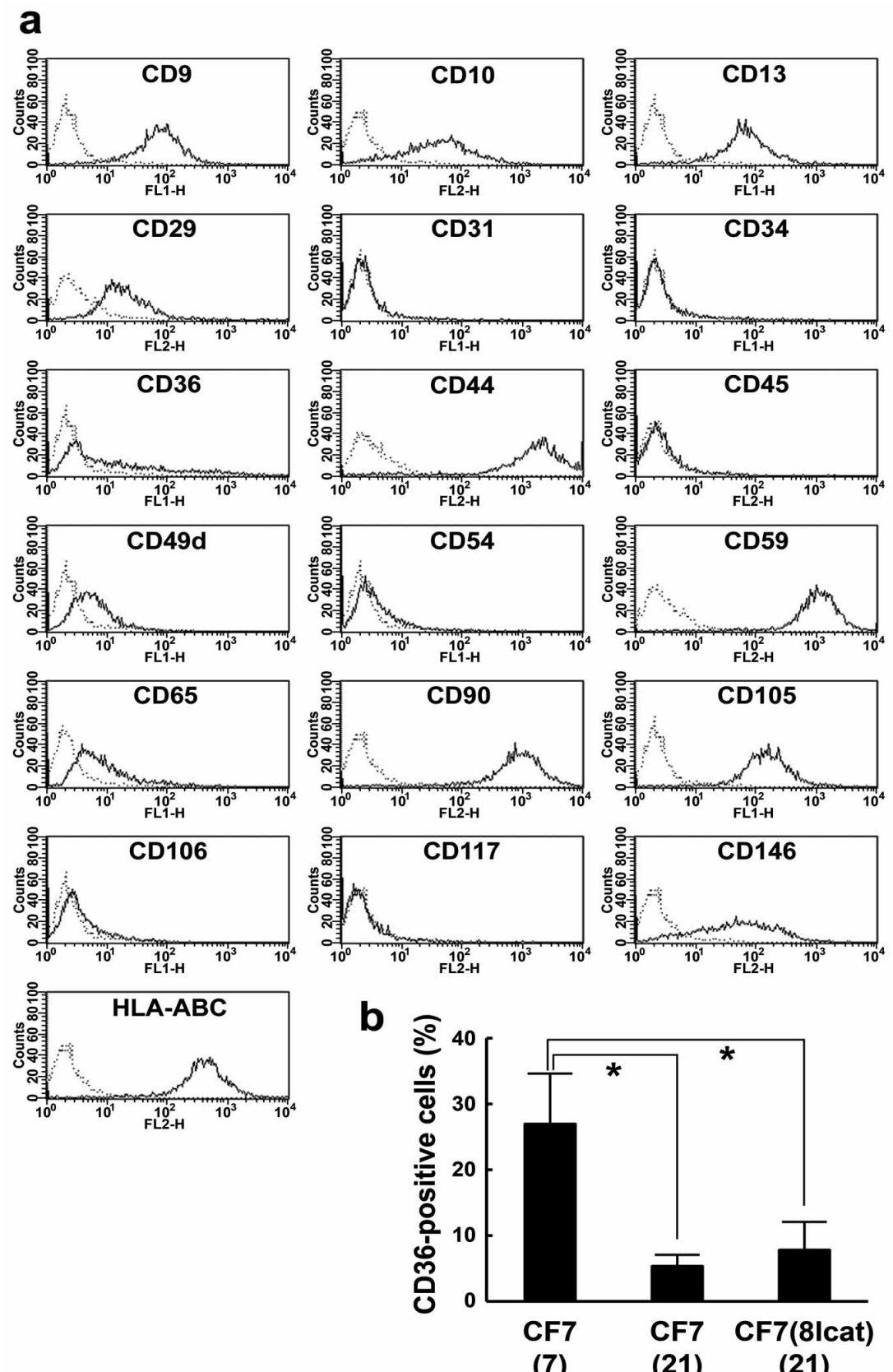

(7)

(21)

(21)

Fig. (2). Cell surface antigen profiles of isolated human ccdPAs by ceiling culture. (a) The cells were harvested at 7 days after ceiling culture, and were immuno-stained with the corresponding antibodies (solid line) or an isotype control (dotted line), and were subjected to a FACS analysis. Histograms for each antibody are presented. (b) CD36-positive cells was examined in the cells harvested from the ceiling culture (CF7(7)), the cells expanded after lcat-gene transduction (CF7(81cat)(21)), and the cells expanded without gene transduction $(\mathrm{CF} 7(21))$. The ratio of CD36-positive cells in the prepared cells is presented as the positive cell rate (\%). Data are presented as the mean \pm SD $(n=3) . * p<0.05$. 
negative for CD31, CD34, CD45, CD54, and CD106. They were moderately positive for CD49d and CD65, and a substantial number of cells were positive for CD36, a marker for adipocytes [25]. The populations of CD36-positive cells after a 14-day in vitro culture of ccdPAs were significantly lower than those at 7 days ( $\mathrm{p}<0.05$, Fig. 2b).

Retroviral Vector-Mediated Gene Transduction and Transduced Gene-Derived Protein Secretion in Human ccdPA

Human ccdPAs were evaluated as a recipient of MoMLV-based gene transduction using various concentrations of the vector and PS with single round of transduction using a ZsGreen-expressing vector. Two types of cells were analyzed, one cell type just after harvesting from the ceiling culture (CF7(7)), while another type was further cultured in the normal manner for an additional week (CF7(14)) in DMEM/FBS. The integrated copy number could be increased to approximately 1.7 and 2.5 copies/cell in CF7(7) and CF7(14) cells, respectively, and a good linear correlation was observed between the integrated copy number and the transduction efficiency (percentage of ZsGreen-positive cells) (Fig. 3a). The transduction efficiency and the integrated copy number were significantly different between the cells of same batch at Days 1 and 2 of gene transduction (Fig. 3b). These results showed that the cells with a higher transduction efficiency of the transduced gene and a lower integrated copy number were obtained by transduction for cells which were seeded and incubated overnight following a 7-day ceiling culture (CF7(8)). The CF7(8) cells were examined as a potential recipient for the human lcat gene. The transduction analyses using the ZsGreen vector showed that a vector concentration of $2.0 \times 10^{9} \mathrm{RNA}$ copies $/ \mathrm{ml}$ resulted in a good correlation between the integrated copy number and ZsGreen-positive cells in two different cell batches (Fig. 3a). The use of the maximum achievable concentration (3.1 x $10^{9}$ RNA copies/ml) of CGT_hLCATRV was compared with that using a concentration of $2.0 \times 10^{9} \mathrm{RNA}$ copies $/ \mathrm{ml}$. Transduction of CF7(8) cells with $3.1 \times 10^{9}$ or $2.0 \times 10^{9}$ RNA copies $/ \mathrm{ml}$ of the vector resulted in no difference in the integrated copy number $(1.65 \pm 0.12$ vs. $1.56 \pm 0.23$ copies/cell). The LCAT protein produced by the lcat gene-
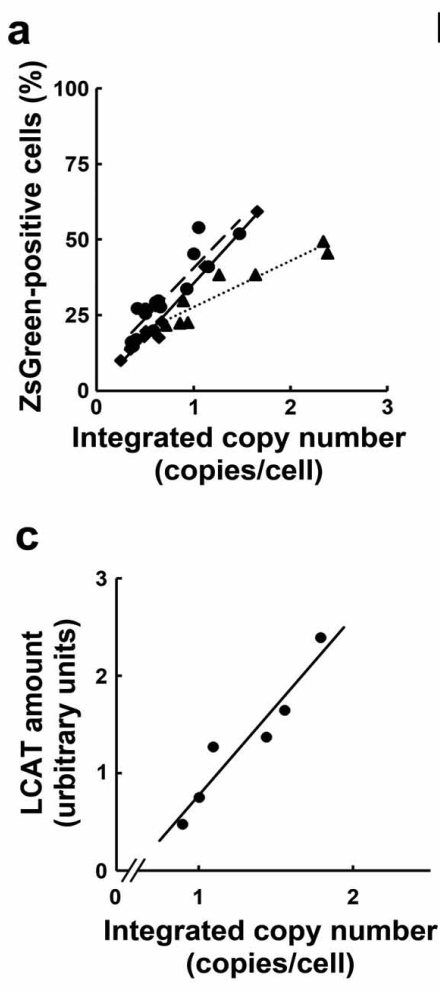
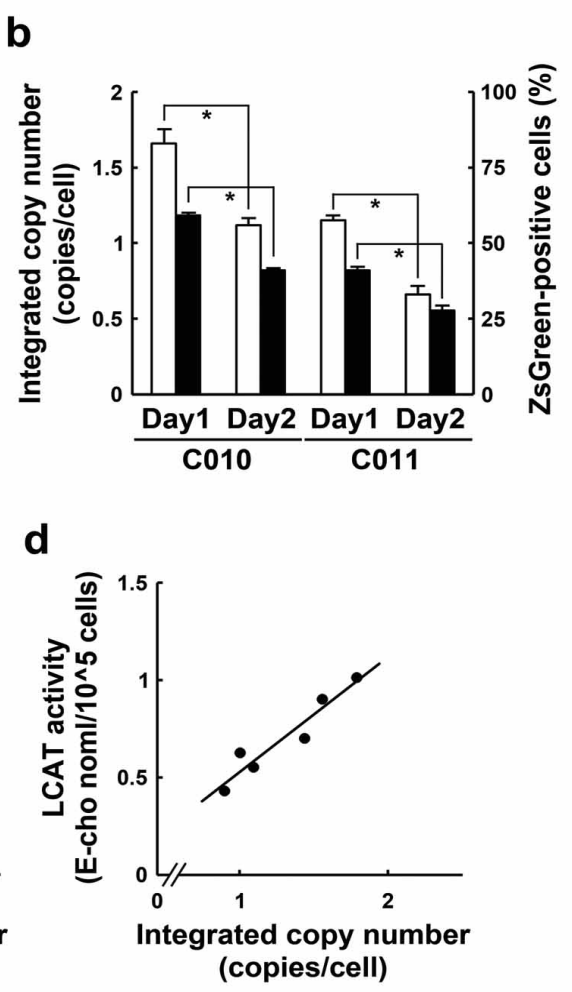

Fig. (3). In vitro evaluation of human ccdPAs as recipients of MoMLV-based retroviral vector-mediated gene transduction and a vehicle for the secretion of functional LCAT protein. (a) Integrated copy number (copies/cell) and ZsGreen-positive cells (\%) were plotted for C010 CF7(7) (closed rhombus), C011 CF7(7) (closed circle), and C011 CF7(14) (closed triangle). Lines are drawn with Pearson rvalues of 0.991, 0.908, and 0.937 for C010 CF7(7) (solid line), C011 CF7(7) (broken line), and C011 CF7(14) (dotted line), respectively ( $\mathrm{p}<0.05)$. (b) Integrated copy numbers (copies/cell, open bars) and ZsGreen-positive cells (\%, closed bars) after a single round of exposure of $2.0 \times 10^{9} \mathrm{RNA}$ copies/ml of virus vector are shown. The cells (C010 and C011) were exposed to the transduction mixture one day (Day 1$)$ or two days (Day 2) after seeding. Data are presented as the mean \pm SD (n=3). ${ }^{*} p<0.05$. (c) Secreted LCAT protein was detected by immunoprecipitation/immunoblotting in culture medium incubated for 3 days with 1 x $10^{5}$ cells (C013). After a densitometric analysis of immunodetected signals for human LCAT protein $(60-65 \mathrm{kDa})$, the integrated copy number and LCAT level (arbitrary units) were plotted (Pearson rvalue of linear coefficient, 0.953, p<0.05). (d) Culture medium incubated with 1 x $10^{5}$ cells (C013) for 3 days were subjected to assay of LCAT activities. The activity was presented by esterified cholesterol production from the cholesterol in the medium of human ccdPAs (Pearson $\mathrm{r}$ value of linear coefficient, $0.954, \mathrm{p}<0.05)$. 
transduced human ccdPAs was analyzed (Fig. 3c and 3d). Seven days after gene transduction, 1 x $10^{5}$ cells were seeded in a 12-well plate, grown for three days, and the supernatant was collected for subsequent assays. LCAT protein production and the LCAT activity were determined by immunoprecipitation/immunoblot (IP-Western) and a cholesterol esterifying assay in the medium, respectively. LCAT protein and activity significantly correlated with the integrated copy number $(\mathrm{r}=0.917$ and 0.954 , respectively, $\mathrm{p}<0.05$ ). Therefore, the activity of the LCAT protein produced by the gene-transduced ccdPA was estimated by the integrated copy number. The lcat gene-transduced ccdPAs produced LCAT protein with a specific activity of $5.2 \pm 0.5 \mathrm{fmol}$ esterified-cholesterol/integrated copy/hr in the culture medium within 3 days.

\section{Properties of the Lcat Gene-Transduced Human ccdPAs during the Manipulation Process}

The effect of in vitro manipulation was evaluated on the ccdPA characteristics regarding adipogenic differentiation ability, expansion rate, cell surface marker expression, transgene stability, and anchorage-independent cell growth. The cells were stimulated to differentiate and Oil Red O staining demonstrated the transduced cells had clearly differentiated into adipocytes (Fig. 4c), and their appearance as well as that without differentiation stimulation, was not obviously different from cells without gene transduction (Fig. 4a-f). The triglyceride contents showed no significant differences between transduced and control cells in C014 samples (1.30 \pm 0.43 vs. $1.25 \pm 0.27 \mathrm{mg} / \mathrm{mg}$ protein). The proliferating cell number and the resultant doubling time were not signifi-
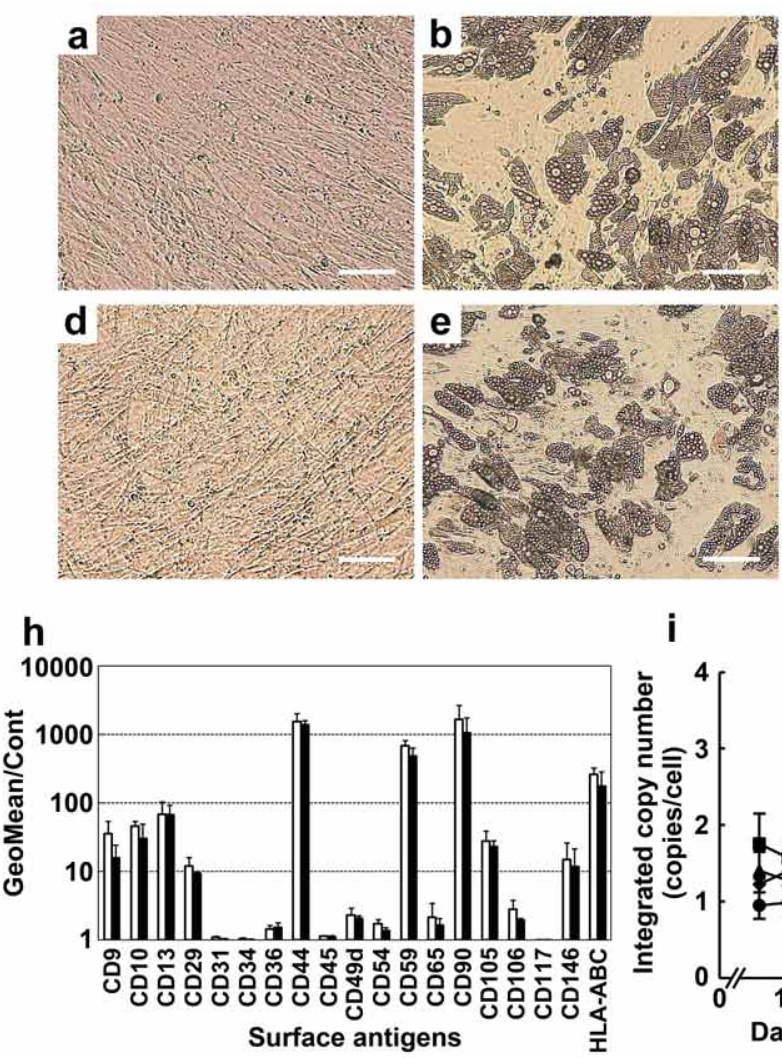
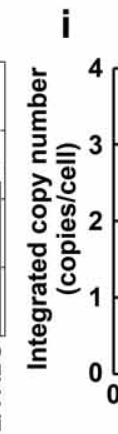

i
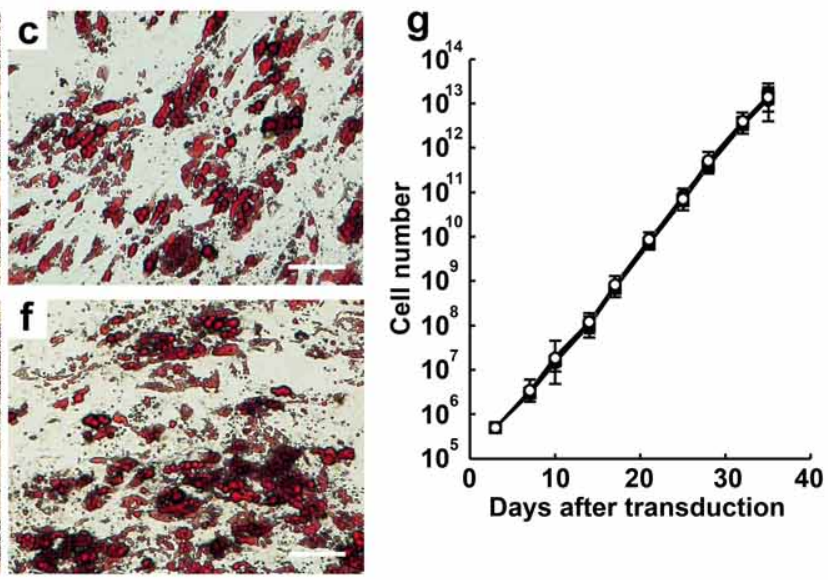

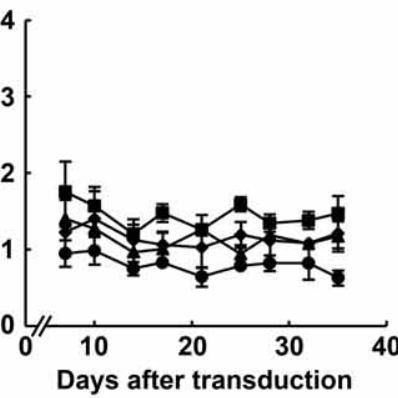

j

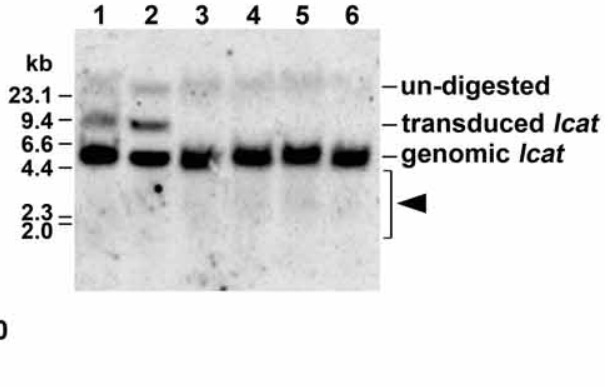

Fig. (4). Characterization of lcat-transduced ccdPA in culture. The lcat-transduced $(\mathbf{a}, \mathbf{b}, \mathbf{c})$ and non-transduced (d, e, f) cells of C013 were incubated for two weeks with $(\mathbf{b}, \mathbf{c}, \mathbf{e}, \mathbf{f})$ or without $(\mathbf{a}, \mathbf{d})$ differentiation stimulation. The appearance of cells was observed with $(\mathbf{c}, \mathbf{f})$ or without (a, b, d, e) Oil Red O staining (magnification bar, $100 \mu \mathrm{m}$ ). (g) C013 cells were transduced, and the resultant cells were passaged. The cell numbers were counted during proliferation for 35 days. The cells were transduced by the conditions of $1.3 \times 10^{9} \mathrm{RNA}$ copies $/ \mathrm{ml}$ on Day 2 (closed circle), $1.3 \times 10^{9} \mathrm{RNA}$ copies/ml on Day 1 (closed triangle), $2.0 \times 10^{9} \mathrm{RNA}$ copies/ml on Day 1 (closed rhombus), or $3.1 \times 10^{9}$ RNA copies/ml on Day 1 (closed square). Doubling times were $32.2 \pm 5.8$ (closed circle), $31.5 \pm 4.0$ (closed triangle), $31.6 \pm 3.9$ (closed rhombus), and $31.3 \pm 4.4$ hrs (closed square), respectively. The doubling time of the control (non-transduced) cells (open circle) was $31.5 \pm$ 4.7 hrs. Data are presented as the mean \pm SD ( $n=3)$. No significant differences were observed in comparison to the control cells. (h) The lcattransduced cells (closed bars) and non-transduced cells (open bars) were expanded in MesenPRO medium for two weeks after gene transduction. The values of Geo/mean for 19 different surface antigens were examined by a flow cytometry analysis. Data are presented as the mean $\pm \mathrm{SD}(\mathrm{n}=3)$. (i) The integrated copy number of lcat-transduced ccdPAs was followed during in vitro culture. Symbols are same as shown in Fig. 4G. Data are presented as the mean \pm SD $(n=3)$. (j) A clonal analysis was performed by Southern blotting in C013 cells. C013 genomic DNA samples were prepared from the cells 18 days after gene transduction. Lanes 1 and 2, lcat gene-transduced clones obtained by transduction of 293 cells; lanes 3, 4, and 5, lcat gene-transduced human ccdPAs with different integrated copy number (lane 3; $0.90 \pm 0.20$; lane 4, $1.65 \pm 0.12$; and lane 5, $1.79 \pm 0.23$ copies/cell); lane 6, non-transduced (control) cells. A smeared faint signal was observed in the lcattransduced ccdPAs (shown by arrow). 
cantly different between the transduced cells and control cells (Fig. 4g). In addition, no significant differences were observed in the cell surface marker expression levels between transduced and control cells (Fig. 4h). The integrated copy number in the transduced ccdPAs was monitored to assess the fate of the transgene during the culture period for 35 days (Fig. 4i). The integrated copy number did not significantly change after gene transduction. A Southern blot analysis using the human lcat gene as a probe revealed that only a faint signal was present independent of the genomic lcat locus, indicating that no amplification of a specific clone had occurred during the expansion process (Fig. 4j). A soft agar assay showed that no anchorage-independent colony formation was present in the gene-transduced human ccdPAs (data not shown). These results demonstrated that the effect of gene transduction was negligible (or denied) on the characteristics of the obtained human ccdPAs regarding the differentiation, cell surface marker expression, transgene stability, and cell growth, in comparison to the non-transduced cells.

\section{Circulating LCAT Supplementation by the Implantation of Lcat Gene-Transduced ccdPA in Mice}

The capacity of human ccdPAs to be recipient cells for lcat gene product delivery was assessed in mice. A cell suspension containing $1.5 \times 10^{6}$ cells was transplanted into the fat tissue of immuno-deficient mice, and the levels of LCAT protein secreted into the serum was determined by the IP-Western method. Human LCAT was clearly detected in the sera of all transplanted mice at Day 1 (Fig. 5a), and was detectable after a month in mice (Fig. 5b). A densitometric analysis revealed that the concentration of human LCAT was approximately $0.26 \pm 0.19 \mu \mathrm{g} / \mathrm{ml}$ at Day 1 . The real-time PCR quantification of the adipose tissue transplanted with lcat-gene-transduced ccdPA showed that the lcat gene was present at $42.9 \pm 27.1 \%$ (Day 1), $1.0 \pm 1.0 \%$ (1 month), and $1.2 \pm 0.7 \%$ (3 months) compared to transplanted cells at Day 0 . These results suggested that approximately $1 \%$ of the lcat gene-transduced ccdPAs survived for 3 months after the transplantation of cells into the fat tissue of mice.

\section{DISCUSSION}

The current study evaluated autologous ccdPAs, the mature adipocye-derived cells, prepared from the subcutaneous fat of patients as a vehicle for therapeutic protein replacement therapy. Adipose tissue contains two major sources of proliferative cell populations, the floating (mature adipocytes) and pellet fractions (SVF), following the centrifugation of collagenase-digested fat tissue. This cell-based gene therapy was developed from the mature adipocyte cultures, since SVF consists of a heterogeneous cell population, including blood cells, fibroblasts, and endothelial cells [15, 16 ] and has some risks in yielding a cell population with an abnormal phenotype in long-term culture in vitro [26, 27]. The ceiling culture of the SVF-removed floating fraction can further enrich the cells derived (or dedifferentiated) from mature adipocytes by the buoyant property of adipocytes during the ceiling culture periods. Our ceiling culture excludes CD31- and CD45-positive cells, and our ccdPAs were negative for CD34, the marker for which adipose-derived stem cells are positive [28-30].

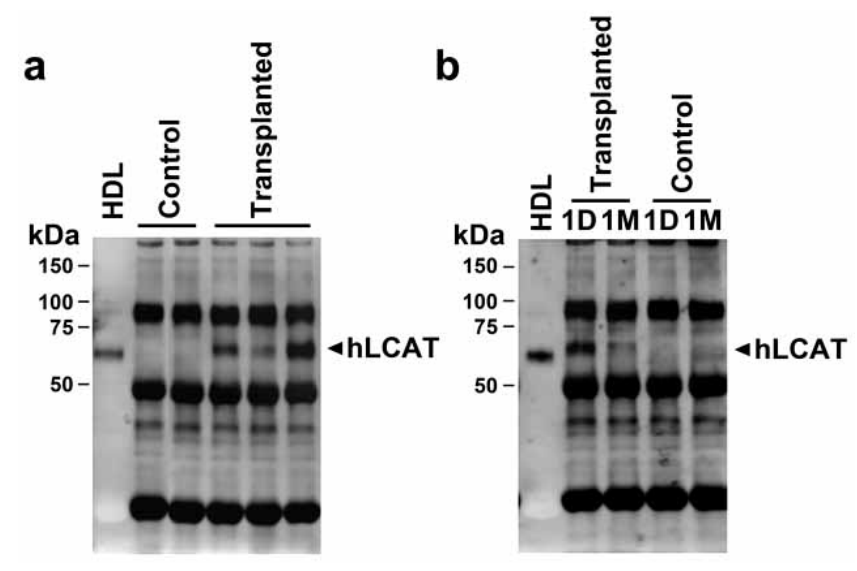

Fig. (5). Circulating human LCAT in NOG mice transplanted with lcat-transduced human ccdPAs. The cell suspension containing $1.5 \times 10^{6}$ lcat-expressing human ccdPA cells (C014, Transplanted) and Ringer's solution containing 0.5\% HSA (Control) were injected into the fat tissue of NOG mice. After one day $(\mathbf{a}, \mathbf{b})$ or one month (b), the mice were sacrificed and serum samples were collected at each time point. D1, next day of injection; M1, 1 month after injection; H, $15 \mu \mathrm{g}$ of HDL (control). At 1 month after transplantation, LCAT was detected in the serum of two mice out of six. At 3 months or later, LCAT was barely detectable in serum (data not shown).

MesenPRO medium, which is optimized for mesenchymal stem cells, provided some advantages in the preparation of ccdPAs through the higher expansion capacity in comparison to DMEM/FBS (Fig. 1b). On the other hand, the MesenPRO medium was less effective for the propagation of human ccdPAs in ceiling culture than DMEM/FBS. Therefore, MesenPRO medium appears to be unsuitable for the proliferation of mature adipocytes in ceiling cultures. The FACS analyses showed that the obtained ccdPAs had a similar profile of surface markers with that of the previously reported adipose-derived cells [31, 32] (Fig. 2a). In addition, the certain population of the ccdPAs retained a mature adipocyte marker (CD36) at an early stage and eventually lost it (Fig. 2b). ccdPA exhibits clearly higher adipogenic potential in comparison to stromal vascular fraction derived cells, commonly used as multi-potential adipose tissue-derived stem cells, suggesting the advanced differentiation levels of ccdPA committed to mature adipocytes (manuscript in preparation). These adipogenic properties are sufficient for the cells to survive in fat tissue and to keep producing therapeutic protein for a long period after transplantation.

Previous reports described mature adipocyte-derived cells that were utilized and evaluated after primary culture for 2 weeks, and these cells were suggested to be a source of regenerative medicine $[31,32]$. We demonstrated that 7 -day primary cultures resulted in substantially better transduction properties than 14-day primary cultures for gene therapy applications. Simple exposure to the viral vector supernatant resulted in a 40-50\% improved transduction efficiency (Fig. 3a and 3b) using ccdPAs after 7-day primary culture, thus suggesting that human ccdPAs serve as an excellent recipient for retroviral vector-based therapeutic applications, in contrast to cell populations in which efficient transduction 
requires drug selection $[3,33]$ or multiple rounds of transduction $[34,35]$. Therefore, a single exposure to $2.0 \times 10^{9}$ RNA copies/ml of CGT_hLCATRV was selected to minimize the transgene copy number in each cell. Furthermore, the transduction efficiency was correlated with the integrated copy number (Fig. 3a and 3b). The lcat-expressing retroviral vector was constructed using pDON-AI, developed by $\mathrm{Yu}$ et al. [36], as a backbone vector. The risk of replicationcompetent retrovirus (RCR) occurrence was minimized by eliminating all the unnecessary structural genes from the MoMLV genome in this vector. In fact, no RCR was detected in the vector preparations (data not shown). The integration sites seemed to be randomly distributed since no clonal expansion was detected by a Southern blot analysis of the transgene following expansion culturing (Fig. 4j), and no increase in the integrated copy number was observed in the preparations (Fig. 4i). No evidence of transformation was observed in the soft agar assay, either at the time of implantation (after three weeks from fat tissue removal) or after long-term extended culture (data not shown). Furthermore, in vivo tumor formation assay by nude mice model revealed no abnormal cell growth after transplantation (unpublished observation). The safety issue of our therapeutic strategy will be carefully evaluated in future clinical studies.

The human lcat gene-transduced ccdPAs yielded the glycosylated LCAT protein (data not shown) that had a molecular weight and in vitro enzymatic activity equivalent to that observed in human serum. An animal study indicated that the human LCAT protein secreted from the implanted transduced human ccdPAs was detectable in blood samples (Fig. 5). The serum of familial LCAT-deficient patients contains less than 10\% LCAT activity compared to that in healthy subjects [11]. Patients with partially inactive LCAT enzymes (8.3-15\% activity of the normal enzyme) have no renal complications [37-39]. Plasma infusion in patients, which raises the plasma LCAT activity level from $9.4 \%$ to $17.4 \%$ compared to normal subjects, resulted in a significant improvement of lipoprotein profiles [13]. These observations suggest that addition of approximately $10 \%$ wild-type LCAT enzyme into patients can prevent the development of the symptoms. The circulating LCAT protein concentration is approximately $6 \mu \mathrm{g} / \mathrm{ml}$ [11] in normal plasma. Transplantation of $1.5 \times 10^{6}$ of lcat-expressing human ccdPAs achieved nearly $5 \%$ of the healthy control level on Day 1 in mice (Fig. 5), but LCAT delivery and cell survival were significantly decreased. Our recent experiments using an autologous mouse transplantation model showed a substantial improvement in LCAT delivery and cell survival (unpublished data), implying that $10^{9}$ cells would yield a therapeutic effect in patients based on the weight ratio between mice and human (1:3000). The fact that the lcat gene-transduced human ccdPAs could be expanded to nearly $10^{10}$ cells within two weeks after gene transduction from $1 \mathrm{~g}$ of fat tissue suggested that human lcat gene-transduced ccdPAs may rescue LCAT deficient patients. Considering the differences in the lipoprotein metabolism between mice and humans, a future strategy to investigate the efficacy of human LCAT replacement therapy may be to establish an in vitro evaluation system employing serum obtained from familial LCATdeficient patients.
In summary, the present study has established a procedure to prepare lcat gene-transduced human ccdPAs for clinical application. These cells have the ability to differentiate into mature adipocytes and secrete functional human LCAT protein. Animal studies revealed that the implanted cells supplied a therapeutic level of LCAT into the serum. Because we confirmed the prolonged secretion of LCAT from lcat-transduced human ccdPAs over three months (data not shown), the significant reduction in LCAT delivery from transplanted cells at one month or later was probably due to the low cell survival rate at the site of transplantation. Therefore, future studies must focus on the improvement of the cell survival rate and prolong the production of the transgene product in vivo.

A clinical trial of an ex vivo gene therapy has shown that the implantation of autologous fibroblasts genetically modified to express human nerve growth factor into the forebrain improved the rate of cognitive decline in subjects with Alzheimer disease [40], indicating that the local delivery of therapeutic protein using autologous fibroblasts as a cell vehicle is clinically relevant. The establishment of clinically applicable procedures for the transplantation of genetransduced human ccdPAs would be useful to obtain further applicable autologous cells for ex vivo gene therapy in patients with serum protein deficiencies who require long-term therapeutic protein supplements. In this study, we have analyzed the LCAT secretion property of lcat gene-transduced ccdPA from healthy volunteers. The propagated cells from different origins showed the LCAT protein secretion enough for our therapeutic strategy. To further expand our therapeutic strategy for the supplementation of other proteins, it is required to evaluate the characteristics of ccdPA from various kinds of fat diseases such as metabolic syndrome which may affect the secretion function of adipose tissues.

\section{ACKNOWLEDGEMENTS}

We thank Atsuo Waki, Kaori Yoshida, Isamu Wakamatsu, Junichi Fukuchi, Kunihiko Ohfuji, Kozo Takamoto, Anna Kakezuka, Mihoko Hatase, Chiho Hattori, Miyuki Ito, Hiroshi Saito, Shohei Akahane, Naomi Kobayashi, Hiromi Harada, Hidemi Sato, Masanori Niimura, Koji Arakawa, Sakura Sekiguchi, Yuko Osa, Koji Fukuda, Reiko Sato, and Rie Ueda, previous members of research division in CellGenTech, Inc., for excellent biochemical analysis and valuable discussions. This study was supported in part by Health and Labour Sciences Research Grants for Translational Research, Japan (H. B.).

\section{REFERENCES}

[1] Reiser J, Zhang XY, Hemenway CS, Mondal D, Pradhan L, La Russa VF. Potential of mesenchymal stem cells in gene therapy approaches for inherited and acquired diseases. Expert Opin Biol Ther 2005; 5 : 1571-84.

[2] Kumar S, Chanda D, Ponnazhagan S. Therapeutic potential of genetically modified mesenchymal stem cells. Gene Ther 2008; 15: 711-5.

[3] Allay JA, Dennis JE, Haynesworth SE, et al. LacZ and interleukin3 expression in vivo after retroviral transduction of marrow-derived human osteogenic mesenchymal progenitors. Hum Gene Ther 1997; 8: 1417-27.

[4] Chuah MK, Van Damme A, Zwinnen H, et al. Long-term persistence of human bone marrow stromal cells transduced with factor VIII-retroviral vectors and transient production of therapeutic lev- 
els of human factor VIII in nonmyeloablated immunodeficient mice. Hum Gene Ther 2000; 11: 729-38.

[5] Lee K, Majumdar MK, Buyaner D, Hendricks JK, Pittenger MF, Mosca JD. Human mesenchymal stem cells maintain transgene expression during expansion and differentiation. Mol Ther 2001; 3 : 857-66.

[6] Krebsbach PH, Zhang K, Malik AK, Kurachi K. Bone marrow stromal cells as a genetic platform for systemic delivery of therapeutic proteins in vivo: human factor IX model. J Gene Med 2003; 5: 11-7.

[7] Van Damme A, Chuah MK, Dell'accio F, et al. Bone marrow mesenchymal cells for haemophilia A gene therapy using retroviral vectors with modified long-terminal repeats. Haemophilia 2003; 9: 94-103.

[8] Kitagawa Y, Bujo H, Takahashi K, et al. Impaired glucose tolerance is accompanied by decreased insulin sensitivity in tissues of mice implanted with cells that overexpress resistin. Diabetologia 2004; 47: 1847-53.

[9] Eliopoulos N, Lejeune L, Martineau D, Galipeau J. Humancompatible collagen matrix for prolonged and reversible systemic delivery of erythropoietin in mice from gene-modified marrow stromal cells. Mol Ther 2004; 10: 741-8.

[10] Ito M, Bujo H, Takahashi K, Arai T, Tanaka I, Saito Y. Implantation of primary cultured adipocytes that secrete insulin modifies blood glucose levels in diabetic mice. Diabetologia 2005; 48: 161420.

[11] Santamarina-Fojo S, Hoeg JM, Assman G, Brewer HB Jr. Lecithin cholesterol acyltransferase deficiency and fish eye disease. In: Scriver CR, Beaudet AL, Sly WS, Valle D, Childs B, Kinzler $\mathrm{KW}$, Volkman BF (eds). In: The metabolic and molecular bases of inherited disease, $8^{\text {th }}$ Ed. (McGraw-Hill Inc., New York) 2001; pp. 2817-33.

[12] Norum KR, Gjone E. The effect of plasma transfusion on the plasma cholesterol esters in patients with familial plasma lecithin: cholesterol acyltransferase deficiency. Scand J Clin Lab Invest 1968; 22: 339-42.

[13] Murayama N, Asano Y, Kato K, et al. Effects of plasma infusion on plasma lipids, apoproteins and plasma enzyme activities in familial lecithin:cholesterol acyltransferase deficiency. Eur J Clin Invest 1984; 14: 122-9.

[14] Casteilla L, Cousin B, Planat-Benard V, Laharrague P, Carmona M, Pénicaud L. Virus-based gene transfer approaches and adipose tissue biology. Curr Gene Ther 2008; 8: 79-87.

[15] Fraser JK, Wulur I, Alfonso Z, Hedrick MH. Fat tissue: an underappreciated source of stem cells for biotechnology. Trends Biotechnol 2006; 24: 150-4.

[16] Gomillion CT, Burg KJ. Stem cells and adipose tissue engineering. Biomaterials 2006; 27: 6052-63.

[17] Zuk PA, Zhu M, Mizuno H, et al. Multilineage cells from human adipose tissue: implications for cell-based therapies. Tissue Eng 2001; 7: 211-28

[18] Gimble J, Guilak F. Adipose-derived adult stem cells: isolation, characterization, and differentiation potential. Cytotherapy 2003; 5: $362-9$.

[19] Gimble JM, Katz AJ, Bunnell BA. Adipose-derived stem cells for regenerative medicine. Circ Res 2007; 100: 1249-60.

[20] Sugihara H, Yonemitsu N, Miyabara S, Yun K. Primary cultures of unilocular fat cells: characteristics of growth in vitro and changes in differentiation properties. Differentiation 1986; 31: 42-9.

[21] Sugihara H, Yonemitsu N, Miyabara S, Toda S. Proliferation of unilocular fat cells in the primary culture. J Lipid Res 1987; 28: 1038-45.
[22] Rogachev VA, Likhacheva A, Vratskikh O, et al. Qualitative and quantitative characteristics of the extracellular DNA delivered to the nucleus of a living cell. Cancer Cell Int 2006; 6: 23

[23] Ishii I, Onozaki R, Takahashi E, et al. Regulation of neutral cholesterol esterase activity by phospholipids containing negative charges in substrate liposome. J Lipid Res 1995; 36: 2303-10.

[24] Ito M, Hiramatsu $\mathrm{H}$, Kobayashi $\mathrm{K}$, et al. $\mathrm{NOD} / \mathrm{SCID} / \gamma_{\mathrm{c}}{ }^{\text {null }}$ mouse: an excellent recipient mouse model for engraftment of human cells. Blood 2002; 100: 3175-82.

[25] Festy F, Hoareau L, Bes-Houtmann S, et al. Surface protein expression between human adipose tissue-derived stromal cells and mature adipocytes. Histochem Cell Biol 2005; 124: 113-21.

[26] Rubio D, Garcia-Castro J, Martín MC, et al. Spontaneous human adult stem cell transformation. Cancer Res 2005; 65: 3035-9.

[27] Ning H, Liu G, Lin G, et al. Identification of an aberrant cell line among human adipose tissue-derived stem cell isolates. Differentiation 2009; 77: 172-80.

[28] Gronthos S, Franklin DM, Leddy HA, Robey PG, Storms RW, Gimble JM. Surface protein characterization of human adipose tissue-derived stromal cells. J Cell Physiol 2001; 189: 54-63.

[29] Zuk PA, Zhu M, Ashjian P, et al. Human adipose tissue is a source of multipotent stem cells. Mol Biol Cell 2002; 13: 4279-95.

[30] Yoshimura K, Shigeura T, Matsumoto D, et al. Characterization of freshly isolated and cultured cells derived from the fatty and fluid portions of liposuction aspirates. J Cell Physiol 2006; 208: 64-76.

[31] Miyazaki T, Kitagawa Y, Toriyama K, Kobori M, Torii S. Isolation of two human fibroblastic cell populations with multiple but distinct potential of mesenchymal differentiation by ceiling culture of mature fat cells from subcutaneous adipose tissue. Differentiation 2005; 73: 69-78.

[32] Matsumoto T, Kano K, Kondo D, et al. Mature adipocyte-derived dedifferentiated fat cells exhibit multilineage potential. J Cell Physiol 2008; 215: 210-22.

[33] Schwarz EJ, Alexander GM, Prockop DJ, Azizi SA. Multipotential marrow stromal cells transduced to produce L-DOPA: engraftment in a rat model of Parkinson disease. Hum Gene Ther 1999; 10: 2539-49.

[34] Chuah MK, Brems H, Vanslembrouck V, Collen D, Vandendriessche $\mathrm{T}$. Bone marrow stromal cells as targets for gene therapy of hemophilia A. Hum Gene Ther 1998; 9: 353-65.

[35] Chiang GG, Rubin HL, Cherington V, et al. Bone marrow stromal cell-mediated gene therapy for hemophilia A: in vitro expression of human factor VIII with high biological activity requires the inclusion of the proteolytic site at amino acid 1648. Hum Gene Ther 1999; 10: 61-76.

[36] Yu SS, Kim JM, Kim S. High efficiency retroviral vectors that contain no viral coding sequences. Gene Ther 2000; 7: 797-804.

[37] Sakuma M, Akanuma Y, Kodama T, et al. Familial plasma lecithin:cholesterol acyltransferase deficiency. A new family with partial LCAT activity. Acta Med Scand 1982; 212: 225-32.

[38] Maeda E, Naka Y, Matozaki T, et al. Lecithin-cholesterol acyltransferase (LCAT) deficiency with a missense mutation in exon 6 of the LCAT gene. Biochem Biophys Res Commun 1991; 178: 460-6.

[39] Gotoda T, Yamada N, Murase T, et al. Differential phenotypic expression by three mutant alleles in familial lecithin:cholesterol acyltransferase deficiency. Lancet 1991; 338: 778-81.

[40] Tuszynski MH, Thal L, Pay M, et al. A phase 1 clinical trial of nerve growth factor gene therapy for Alzheimer disease. Nat Med 2005 ; $11: 551-5$ 